\title{
Guest editorial: Special issue on device-to-device service and network management for beyond 4G mobile networks
}

\author{
Young-June Choi ${ }^{1} \cdot$ Alexander W. Min ${ }^{2} \cdot{\text { Zhetao } \mathbf{L i}^{3}}^{3}$
}

Published online: 12 March 2016

(C) Springer Science+Business Media New York 2016

Device-to-device (D2D) communications refer to the technologies that enable devices to communicate directly without the intervention of a base station (e.g., eNodeB in LTE). D2D communication scenarios include public safety as well as proximity services, such as social networking and advertisement for by-passers. For cellular networks, D2D can improve spectrum utilization, offload traffic for the base station, and save energy of mobile devices. In Releases 12 and 13 of the 3rd Generation Partnership Project (3GPP), D2D is being standardized to support peer discovery and direct communication, which include peer synchronization and new channel definitions. However, research issues are not limited to the scope of such standards. For $5 \mathrm{G}$ networks, we need to tackle new topics of spectrum sharing, duplexing, interference management, and extension to vehicle-to-everything (V2X).

The goal of this special issue is to present, exchange, and discuss the most recent advances in various aspects of the D2D solutions and provide non-precedent services to the end users with emerging technologies. The detailed topics of the special issue include D2D service architecture for $5 \mathrm{G}$ networks, D2D neighbor discovery, D2D radio resource

Young-June Choi

choiyj@ajou.ac.kr

Alexander W. Min

alexander.w.min@intel.com

Zhetao Li

liztchina@hotmail.com

1 Ajou University, Yeongtong-gu, Suwon, Korea

2 Intel Labs, Hillsboro, OR, USA

3 Xiangtan University, Xiangtan, China management, D2D mobility management, D2D interference management and D2D clustering. After a rigorous review process, we have selected six papers which cover those topics.

The paper "Novel Power Control and Collision Resolution Scheme for Device-to-Device Discovery," authored by Jongwoo Hong, Seungil Park, and Sunghyun Choi, addresses the problem of reducing interference for D2D communications and improving neighboring device discovery in distributed D2D communications environments without a centralized controller. The authors identify interference and device discovery as two key challenges in D2D communications, and propose novel distributed power control and collision resolution schemes to enable more efficient D2D communications without adding much complexity to the system. The proposed schemes are evaluated with simulations and demonstrate significant performance benefits in terms of distributed discovery success ratio compared to the conventional schemes. The proposed schemes can be applied to various D2D communication scenarios and applications in LTE-A and future 5G networks with mass deployment of mobile and machine-type devices.

The paper "Underlaid-D2D-assisted Cooperative Multicast based on Social Networks," authored by Wenjun $\mathrm{Xu}$, Shengyu Li, Yue $\mathrm{Xu}$, and Xuehong Lin, proposes a new cooperative multicast scheme that maximizes the total multicast rate with improved resource utilization. The proposed schemes leverage D2D communications for data forwarding and leverage social connections in relay user selection where strong social connections are considered to be an indication of incentive for cooperative relay. The authors formulate the problem as an optimization problem to maximize the overall multicast rate, and employee a two-step approach for the relay user selection and transmit power control. The proposed schemes are evaluated 
with simulations in realistic scenarios, demonstrating up to $85 \%$ performance gain in multicast rate compared to the conventional cooperative multicast approaches.

The paper "Utility-aware Data Transmission Scheme for Delay Tolerant Networks," authored by Fu Xiao, Xiaohui Xie, Zhifei Jiang, Lijuan Sun, and Ruchuan Wang, focuses on routing approaches in delay-tolerant networks and proposes a utility-aware data transmission scheme which considers both internal property and external contact of nodes. This scheme sets a central group and chooses the appropriate number of nodes with higher utility for message forwarding which is supported by utility-aware router (UAR). The authors further present two types of UAR: probimproved UAR and buffer-improved UAR to reduce the overhead.

The paper "Device-to-Device Resource Allocation in LTE-Advanced Networks by Hybrid Particle Swarm Optimization and Genetic Algorithm," authored by Shijie Sun, Kwang-Yul Kim, Oh-Soon Shin, and Yoan Shin, aims at reducing interference for cellular users and allocating frequency resources for D2D pairs. By exploiting Particle Swarm Optimization (PSO) and Generic Algorithm (GA), the authors propose hybrid PSO-GA based resource allocation schemes that maximize system throughput by allowing up to two D2D pairs to share the same frequency resource with one cellular user equipment (CUE). They also present a scheme to mitigate the interference caused to CUEs.

The paper "Cooperative Device Discovery for MultiInterface Self-Organizing Networks," authored by Do-yun Kim and Young-June Choi, presents a realistic device discovery scheme for multiple-interfaced hierarchical networks. The authors extend the existing jump-and-stay rendezvous algorithm to have two types of interfaces: standby and scan, to improve the time-to-rendezvous for hierarchical networks. They also propose a cooperative rendezvous algorithm that achieves better performance compared to conventional ones.

The paper "Optimization of Resource Allocation for Underlay Device-to-Device Communications in Cellular Networks," authored by Hieu V. Nguyen, Quang Duong, Van-Dinh Nguyen, Yoan Shin, and Oh-Soon Shin, improves the spectral efficiency of cellular systems and meets the growing demand for wireless local services. However, it is very difficult to get the whole channel state information (CSI) on the interference channels and the desired channels. The authors propose D2D resource allocation schemes based on distance as cost functions between nodes. Two optimization problems are formulated: a linear sum assignment problem (LSAP) and a linear bottleneck assignment problem (LBAP). The effectiveness of the proposed algorithms is shown via numerical results.

Finally, we would like to thank all the authors for their valuable contributions for this special issue. Also, we would like to extend our gratitude to the Editor-in-Chief, Prof. Xuemin (Sherman) Shen as well as Ms. Melissa Fearon, Ms. Jenilyn Jaos, and Mr. Alberto Bactong from the Editorial Office for helping us publish this special issue.

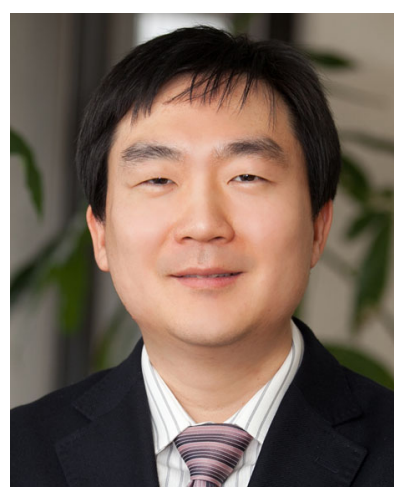

Young-June Choi is an associate professor at Ajou University, Korea. He received his B.S., M.S., and Ph.D. degrees from the Department of Electrical Engineering and Computer Science, Seoul National University, Korea, in 2000, 2002, and 2006, respectively. From Sept. 2006 through July 2007, he was a postdoctoral researcher at the University of Michigan, Ann Arbor, MI, USA. From 2007 through 2009, he was with NEC Laboratories America, Princeton, NJ, USA, as research staff member. He joined Ajou University from Sept. 2009 as a faculty member. He is an adjunct professor at Seoul National University from Sept. 2015 and a honorary professor at Xiangtan University, China from Dec. 2013. He has served as an organizing committee member for IEEE Secon 2012, IEEE VTC Spring 2014, IEEE ICUFN 2013, 2014, 2015, 2016, IEEE ICTC 2014, 2015, 2016, and many other conferences. He has been also serving as editor of Journal of Communication and Networks since 2011, editor of ICT Express since 2015, and associate editor of Elsevier Digital Communications and Networks since 2015. He was awarded Haedong Young Researcher Award from Korea Information and Communication Society in 2015 and Gold Prize from Samsung Humantech Thesis Contest in 2006. He has published more than 100 papers and holds about 30 patents in the area of mobile networks, radio resource management, and dynamic spectrum access.

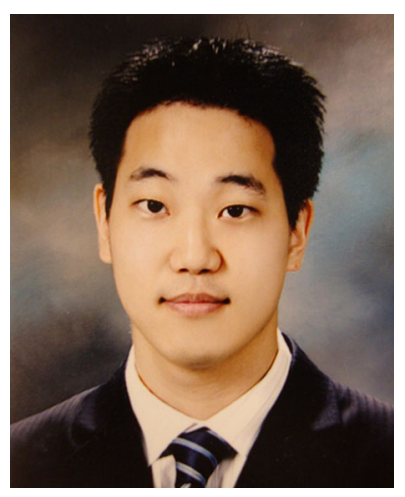

Alexander W. Min is a Research Scientist in Wireless Communications Research at Intel Labs. His research interests include next generation wireless systems, low-power mobile platforms, softwaredefined networking, network virtualization, and wireless connectivity for wearable and IoT devices. He received the B.S. degree in Electrical Engineering from Seoul National University, Korea, in 2005, and the M.S. and Ph.D. degree in Electrical Engineering and Computer Science from the University of Michigan, Ann Arbor, in 2007 and 2011. He has published 25+ technical papers in the area of wireless communications and mobile computing and holds 40+ patents issued/filed. He has served on technical program committees for leading conferences including the IEEE Infocom 2014-2016. 


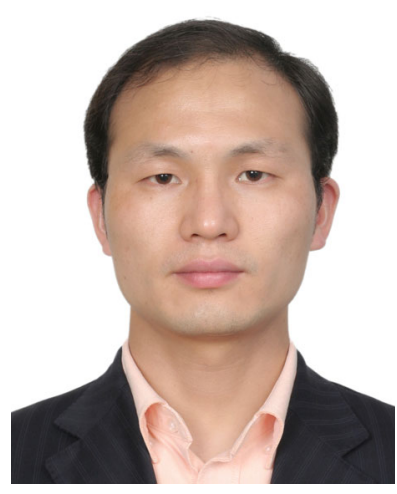

Zhetao $\mathbf{L i}$ is an associate professor in College of Information Engineering, Xiangtan University, China. $\mathrm{He}$ was born in Hunan province, P.R.China. He received the B.Eng. degree in Electrical Information Engineering from Xiangtan University in 2002, the M.Eng. degree in Pattern Recognition and Intelligent System from Beihang University in 2005, and the Ph.D. degree in Computer Application Technology from Hunan University in 2010. Dr. Li was a visiting researcher at Ajou University from May to Aug 2012. From Dec 2013 to Dec 2014, he was a post-doc in wireless network at Stony Brook University. From Dec 2014 to Mar 2015, he is a visiting professor at Ajou University. His research interests include wireless communication and signal processing. His current researches focus on cognitive network, the 4G mobile communication networks, compressive sensing, and distributed data collection. He has published about thirty papers and submitted about forty patents on wireless network and compressive sensing in the past five years. For his successes in teaching and research he received the Second Prize of Fok Ying Tung Education Foundation Fourteenth Young Teachers Award in 2014. 